\title{
Generator Effects on the Optimal Control of a Power Assisted Diesel-Electric Powertrain
}

\author{
Martin Sivertsson and Lars Eriksson \\ Vehicular Systems, Dept. of Electrical Engineering \\ Linköping University, SE-581 83 Linköping, Sweden \\ Email:\{marsi,larer\}@isy.liu.se
}

\begin{abstract}
Optimal control of a diesel-electric powertrain in transient operation is studied. The attention is on how generator limits affect the solution, as well as how the addition of a small energy storage can assist in the transients. Two different types of problems are solved, minimum fuel and minimum time, with different generator limits as well as with and without an extra energy storage. In the optimization both the output power and engine speed are free variables. For this aim a 4-state mean value engine model is used together with models for the generator and energy storage losses. The considered transients are steps from idle to target power with different amounts of freedom, defined as requirements on produced energy, before the requested power has to be met. For minimum fuel transients the energy storage remains virtually unused for all requested energies, for minimum time it does not. The generator limits are found to have the biggest impact on the fuel economy, whereas an energy storage could significantly reduce the response time.
\end{abstract}

\section{INTRODUCTION}

In off-highway applications the driving profiles are often very transient. In such applications the diesel-electric powertrain, such as the BAE Systems TorqE ${ }^{\mathrm{TM}}$, see Fig. 1, offers the potential to increase the performance and lower the fuel consumption, due to the lack of mechanical link between the diesel engine and the wheels. Through this electrification of the powertrain the engine speed can be chosen freely which also enables the powertrain to produce maximum power from standstill. This in combination with the torque characteristics of the electric motors can thus increase performance and potentially lower fuel consumption, especially in transient operation.

In previous papers it is studied how to best take advantage of the extra freedom available in the diesel-electric powertrain, see [1], [2], and in [3] those results are extended by including a model for the generator losses as well as a small energy storage. In other related articles on optimal transient control of diesel-engines different optimization methods are used to minimize pollutants during transient operation for known engine speeds, see for instance [4] or, as in [5] the optimal engine operating point trajectory for a known engine power output trajectory is derived. The diesel engine is modeled as an inertia with a Willans-line efficiency model and the optimal solution is found using dynamic programming and Pontryagins maximum principle. Due to the complexity of the non-linear model used in this paper such methods aren't feasible. Instead the problem is solved using Tomlab/PROPT which uses

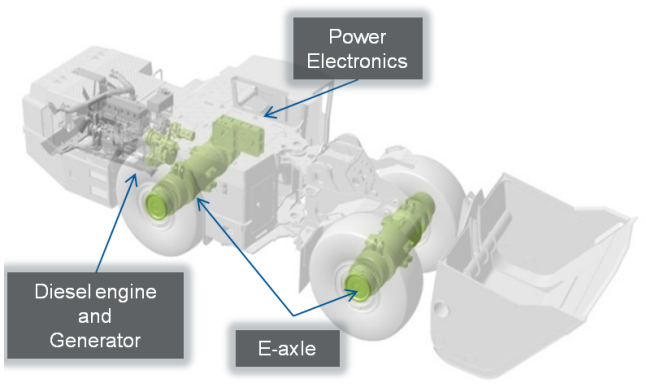

Fig. 1. BAE Systems TorqE $E^{\mathrm{TM}}$ powertrain.

pesudospectral collocation methods to solve optimal control problems.

The contribution of this paper is the study of the effects the generator limits have on the optimal control from idle to a target energy for two different criteria with the engine output power and engine speed considered free variables during the transient, with and without a short term storage to assist in the transient. To also be able to study how large the energy storage should be, the size is not fixed. A nonlinear, four state, four input mean value engine model (MVEM) is used in the study together with models for the losses in the generator and energy storage. The MVEM incorporates the turbocharger dynamics as well as the nonlinear multiple input multiple output nature of the diesel engine. The model is implemented with continuous derivatives to facilitate analytical derivatives during the numerical solution of the optimal control problem.

\section{MODEL}

The modeled powertrain consists of a 6-cylinder 12.7-liter SCANIA diesel engine with a fixed-geometry turbine and a wastegate for boost control, equipped with a generator and energy storage. The states of the MVEM are engine speed, $\omega_{i c e}$, inlet manifold pressure, $p_{i m}$, exhaust manifold pressure, $p_{e m}$, turbocharger speed, $\omega_{t c}$, charge in the energy storage, $q$, and produced energy of the powertrain, $E_{g e n}$. The controls are injected fuel mass, $u_{f}$, wastegate position, $u_{w g}$, generator power, $P_{g e n}$, and power from the energy storage, $P_{b a t t}$. The engine model consists of two control volumes, intake and exhaust manifold, and four restrictions, compressor, engine, turbine, and wastegate. The control volumes are modeled with 


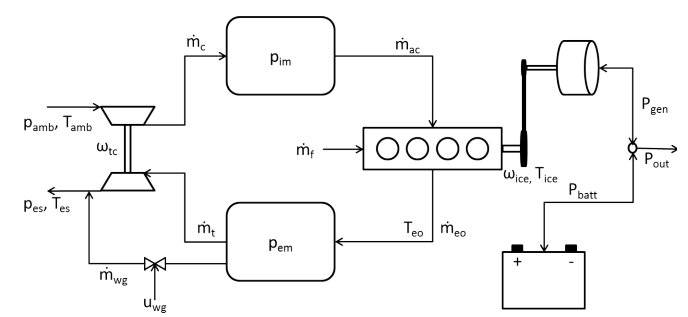

Fig. 2. Structure of the MVEM. The modeled components as well as the connection between them.

the standard isothermal model, using the ideal gas law and mass conservation. The engine and turbocharger speeds are modeled using Newton's second law. The governing differential equations of the MVEM are:

$$
\begin{aligned}
\frac{d \omega_{i c e}}{d t} & =\frac{1}{J_{g e n s e t}}\left(T_{i c e}-\frac{P_{\text {mech }}}{\omega_{i c e}}\right) \\
\frac{d p_{i m}}{d t} & =\frac{R_{a} T_{i m}}{V_{i s}}\left(\dot{m}_{c}-\dot{m}_{a c}\right) \\
\frac{d p_{e m}}{d t} & =\frac{R_{e} T_{e m}}{V_{e m}}\left(\dot{m}_{a c}+\dot{m}_{f}-\dot{m}_{t}-\dot{m}_{w g}\right) \\
\frac{d \omega_{t c}}{d t} & =\frac{P_{t}-P_{c}}{\omega_{t c} J_{t c}}-w_{f r i c} \omega_{t c}^{2}
\end{aligned}
$$

The model used is a modified version of the model presented in [6]. The submodels as well as the parameters and constants used can be found in [1]. As in [3], the model is also augmented with a model for the generator losses as well as a model for the energy storage.

\section{Problem Formulation}

The considered problem in this paper is a step from idle to a requested output power and energy. In order to evaluate the potential of the diesel-electric powertrain on this type of transient, two optimal control problems are formulated, minimum time and minimum fuel, as follows:

$$
\begin{array}{ll}
\min & \int_{0}^{T} \dot{m}_{f} \mathrm{dt} \quad \text { or } \quad \min T \\
\text { s.t. } & \dot{x}=f(x, u),
\end{array}
$$

where $x$ is the states of the MVEM and $\dot{x}$ is defined by (1)(4). The studied transients are subject to constraints imposed by the components, such as maximum torque and minimum speed, as well as environmental constraints, i.e. a limit on $\phi_{\lambda}$ set by the smoke-limiter, and also a requirement that the control has to end in a stationary point. The constraints are:

$$
\begin{array}{ll}
x(0)=\text { idle }, & \dot{x}(T)=0 \\
T_{i c e} \leq T_{i c e, \text { max }}\left(\omega_{\text {ice }}\right), & \omega_{\text {ice }} \geq \omega_{\text {ice } \text { min }} \\
\phi_{\lambda} \geq 0, & P_{\text {out }}=P_{\text {batt }}+P_{\text {gen }} \\
0 \leq P_{\text {out }} \leq P_{\text {req }}, & P_{\text {out }}(T)=P_{\text {req }} \\
P_{\text {batt }}=0 \text { or } P_{\text {batt }}(T)=0, & P_{\text {mech }}=P_{\text {gen }}+P_{\text {loss }} \\
\int_{0}^{T} I_{\text {batt }} d t=0, & \int_{0}^{T} P_{\text {out }} d t=E_{\text {req }}
\end{array}
$$

The problem in (5)-(6) is how to control the diesel-electric powertrain in order to be able to satisfy the operators power and energy request, either as fast as possible, or as fuel efficient as possible, where $E_{r e q}$ can be interpreted as a measure on the amount of freedom given to the powertrain, in terms of produced energy, before the operators power request has to be met. The generator has two limits, one for continuous operation and one for peak power. In order to study how these constraints affect the solution the problem is solved for four different cases. The cases are listed in Table I.

\section{TABLE I}

THE DIFFERENT GENERATOR LIMITS USED.

$$
\begin{aligned}
& \text { Standard: } \quad P_{\text {mot, peak }} \leq P_{\text {mech }} \leq P_{\text {gen }, \text { peak }} \\
& \text { Cont-lim: } \quad P_{\text {mot,cont }} \leq P_{\text {mech }}(T) \leq P_{\text {gen, con }} \\
& \text { Peak-lim: } \quad P_{\text {mot,cont }} \leq P_{\text {mech }} \leq P_{\text {gen }, \text { cont }} \\
& \text { Power-lim: } \quad-300 k W \leq P_{\text {gen }} \leq 300 k W
\end{aligned}
$$

In the first case the generator is allowed to exceed the continuous limit, but not the peak, and also has to end in a stationary point below the continuous limit, in the second case the generator is never allowed to exceed the continuous limit, in the third case it is only limited by the peak limit, and in the fourth only limits of the power electronics are enforced. The different limits as well as the maximum torque line can be seen in Fig. 7.

In order to study the effects of adding a small energy storage to assist during the transients the problem is solved with both $P_{b a t t}=0$ and with $P_{b a t t}$ as a free variable. In order to ensure stationarity in charge, $q$, in the final time step $P_{b a t t}(T)=0$ in both cases.

\section{NUMERICAL SOLUTION PATH}

To avoid oscillating solutions the same method as in [3] is used. The integral of the squared state derivatives is added to the cost function with a weighting factor that is iteratively decreased, and the lowest weight that gives a smooth solution is then used. The worst case change from this technique is less than $0.15 \%$ in fuel consumption and $0.4 \%$ in time. As also discussed in [3] the minimum time solution above a certain $E_{r e q}$ is not unique, due to that the output power is limited below the maximum power of the engine. To handle this time is first minimized and then a second problem is solved where fuel is minimized, according to the strategy previously discussed, with $T \leq \min T+\epsilon$, where $\epsilon$ means that the minimum time is rounded up. How much the time needs to be rounded up to obtain a smooth solution differs slightly, but the largest increase in duration from this technique is less than a tenth of a permil. The obtained solution is both smooth and with lower fuel consumption without any significant effects on the duration.

\section{POWER TRANSIENTS}

In order to benchmark the solutions to the problem defined in Section III, the problem is first solved without requirements on produced energy for the different cases, for the two criteria, with and without energy storage. The problem then becomes a step from idle to a terminal power, $P_{r e q}=170 \mathrm{~kW}$. Since the solutions to the power transients don't have the problems discussed in Section IV those techniques are not used. The 
results are shown in Fig. 3. There it is seen that the results from [1] hold even when a model for the generator losses is added. The main characteristic of the solution is more dictated by the maximum torque-line and the smoke-limiter than by the efficiency of the engine, but the end point and how it is approached differ. Whereas the minimum time solution follows the smoke-limiter until the end, the minimum fuel solution ends with cutting fuel as the stationary point is approached. Then the wastegate is actuated to get stationarity. The different generator limits however affect the solution, and this will be discussed below.

\section{A. Standard and Continuous-lim}

A difference between the two criteria is how the energy storage is used. The minimum time solution uses the generator in motoring mode for the first $0.15 \mathrm{~s}$, accelerating the engine, and thus increasing the backpressure and consequently turbocharger speed. It then switches to generating mode, recharging the energy storage. The minimum fuel solution switches operating mode for the generator three times. First it uses the generator in motoring mode, accelerating the engine. It then goes over to charging the energy storage, charging it to a level over zero, a buffer later used to assist in the acceleration towards the end of the transient.

In Table II the change in time and fuel consumption compared to $\min T, P_{b a t t}=0$, Standard-lim, which is used as a baseline throughout the paper, is shown. Without the use of an energy storage the minimum fuel uses $3 \%$ less fuel than the minimum time, however this comes at the price of a $2 \%$ time increase. Adding an energy storage has only slight effects on the fuel economy, however the time duration decreases so the minimum fuel solution is actually faster than the baseline. The biggest effects can be seen when time is minimized, the time consumption decreases with $11 \%$ but at a price of $4 \%$ increase in fuel consumption.

\section{B. Peak and Power-lim}

The minimum time results are the same as with Standardlim, which is to be expected since the results for Standardlim never exceed the continuous limit. For minimum fuel the solution is to accelerate the engine to a higher engine speed and then decelerate towards the stationary point. This exploits the inertias in the system building more kinetic energy in the turbocharger while reducing the kinetic energy of the engine in the final operating point, thus increasing the efficiency. With energy storage the fuel is cut in a similar manner as without energy storage but the generator is also used, charging the energy storage. This extra energy is instead used in the beginning of the transient to accelerate the engine. Since none of the solutions exceed the peak limit, Peak-lim and Power-lim both produce the same solution.

In Table II the results for a step in power are compared to those for Standard-lim. Since the minimum time solution never exceeds the continuous limit the results are the same. The minimum time solution with energy storage is however $16 \%$ faster and with only a $0.2 \%$ increase in fuel, an improvement
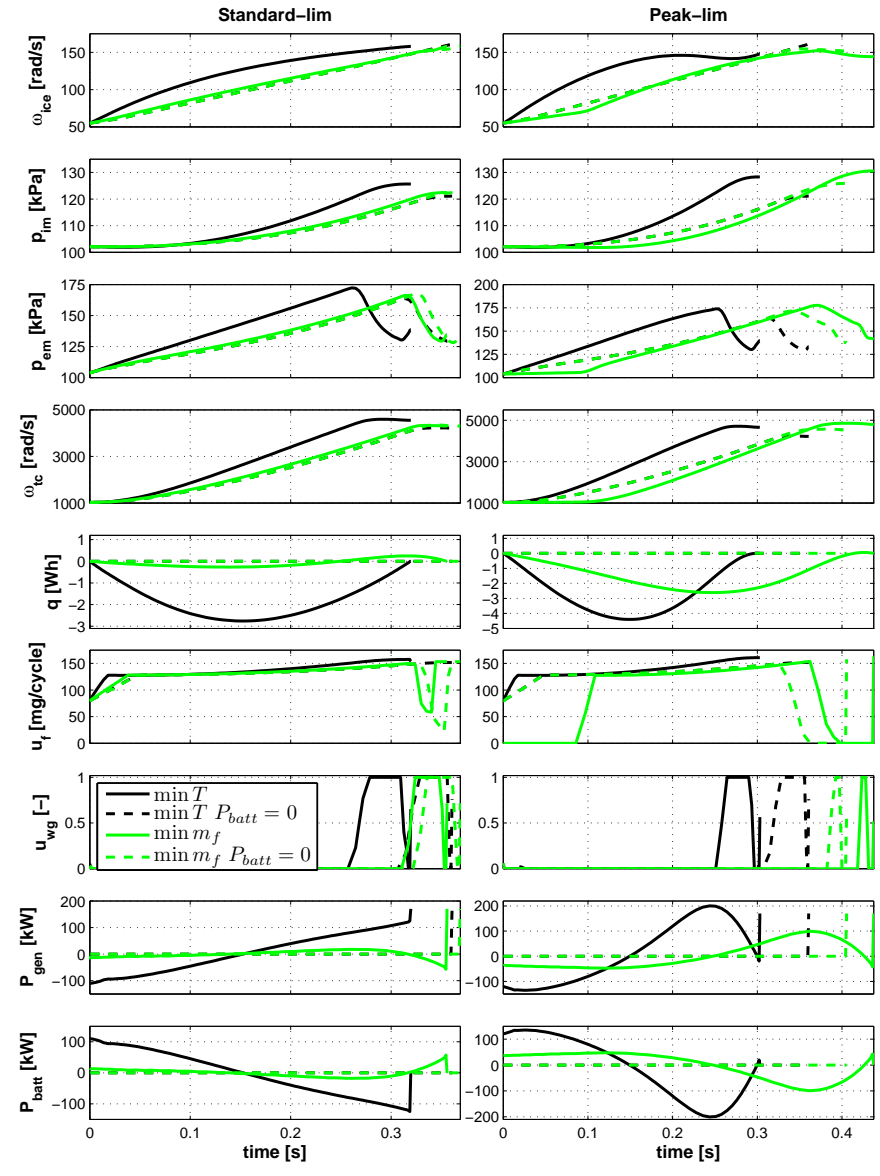

Fig. 3. States and controls for a step from idle to target power for minimum time and minimum fuel, with and without assistance from the energy storage.

TABLE II

CHANGE IN TIME AND FUEL CONSUMPTION FOR A POWER STEP WITH THE ADDITION OF A SMALL ENERGY STORAGE. ALL RESUlTS RELATIVE $\min T, P_{b a t t}=0$, STANDARD-LIM.

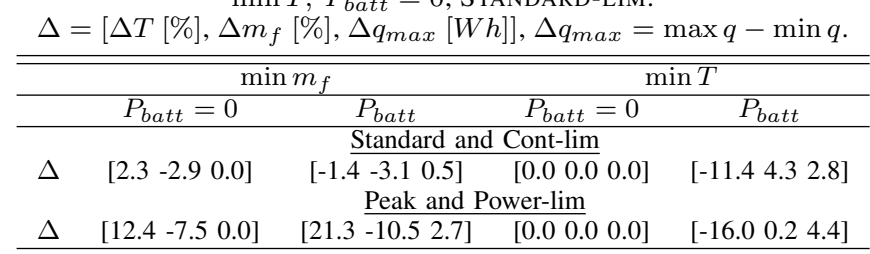

of $4.6 \%$ and $4.1 \%$ respectively, compared to Standard-lim. For minimum fuel the increase in fuel economy is $7.5 \%$ without, and $10.5 \%$ with energy storage and the increase in duration is $12.4 \%$ and $21.3 \%$ respectively. This means that just by being allowed to exceed the continuous limit the fuel economy increases by $4.6 \%$, and with an energy storage this increases another $3 \%$, however at the cost of increased duration.

\section{ENERGY TRANSIENTS}

The problem defined in Section III is solved with $P_{r e q}=$ $170 \mathrm{~kW}$ for three different $E_{\text {req }}$ with the different generator limits, with and without the use of an energy storage. The results are shown in Fig. 4-6. For comparison between the different cases see Fig. 7-8. Due to page limitations the state and control trajectories for Peak-lim are left out. 

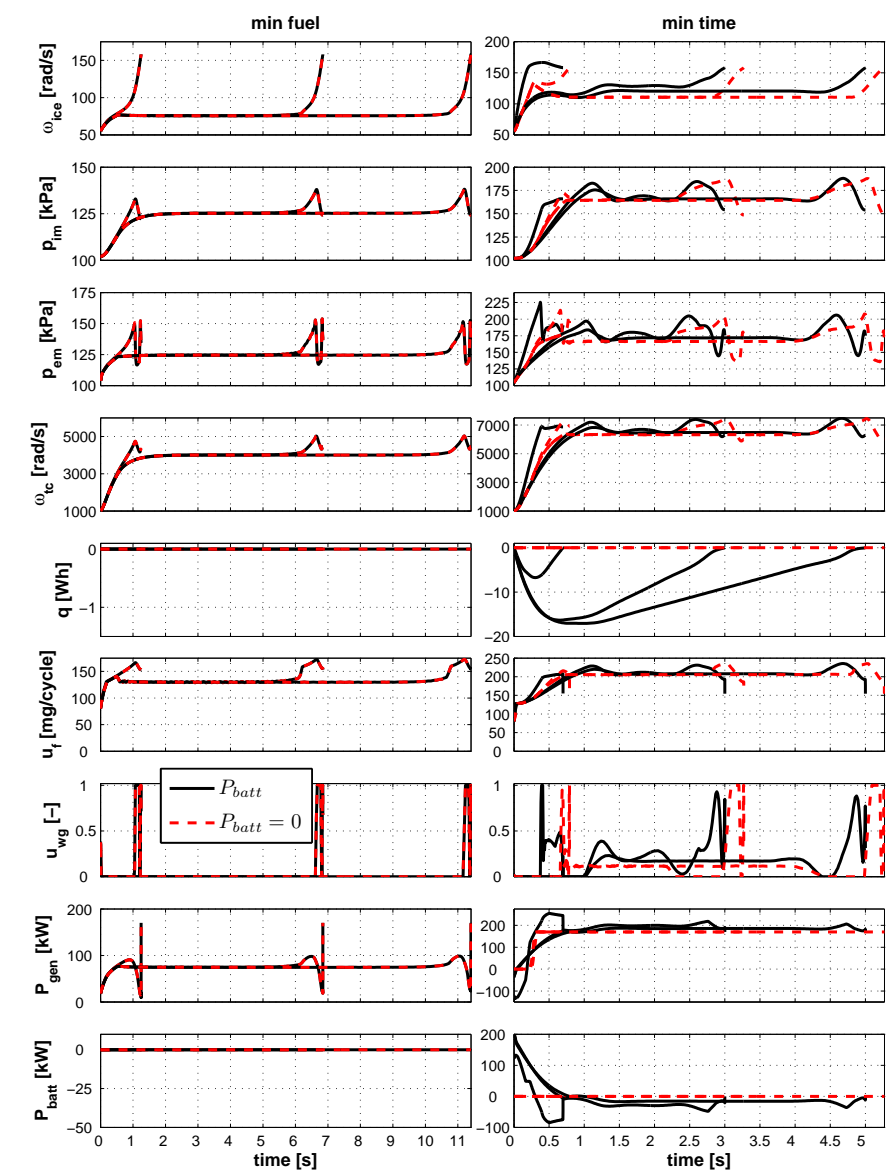

Fig. 4. Solutions for $E_{r e q}=\left[\begin{array}{l}85 \\ 510\end{array} 850\right] \mathrm{kJ}$ with and without $P_{\text {batt }}=0$ Standard-lim.

\section{A. Minimum fuel}

The minimum fuel solutions all have the same characteristic. The control is to accelerate, following the smoke-limit and maximum torque line, towards the region of peak efficiency for the generator and since this is below the continuous limit this applies to all the cases. Then if $E_{r e q}$ is large enough the control has a stationary phase at this point before the engine is accelerated to the region with highest efficiency that also fulfills the end constraints for the given case. The transient ends with control actuation to bring the state derivatives to zero. The only exceptions are $E_{r e q}=85 \mathrm{~kJ}$ for Peak and Power-lim. This is since $E_{r e q}$ is so small so the solution is mainly dictated by fulfilling the end constraints. Instead the engine is accelerated to a higher engine speed and then braked by the generator, converting the kinetic energy to output power. Standard-lim and Cont-lim are very similar, as well as Peak and Power-lim. For Standard and Cont-lim this is because even though allowed to, the generator hardly exceeds the continuous limit, so the difference is just during the periods when the continuous limit is exceeded the generator instead follows it. For Peak and Power-lim this similarity is due to that their peak efficiency regions for $P_{r e q}$ are close. None of the solutions with Standard and Cont-lim use the energy storage, instead the solution with energy storage is the same as without. With
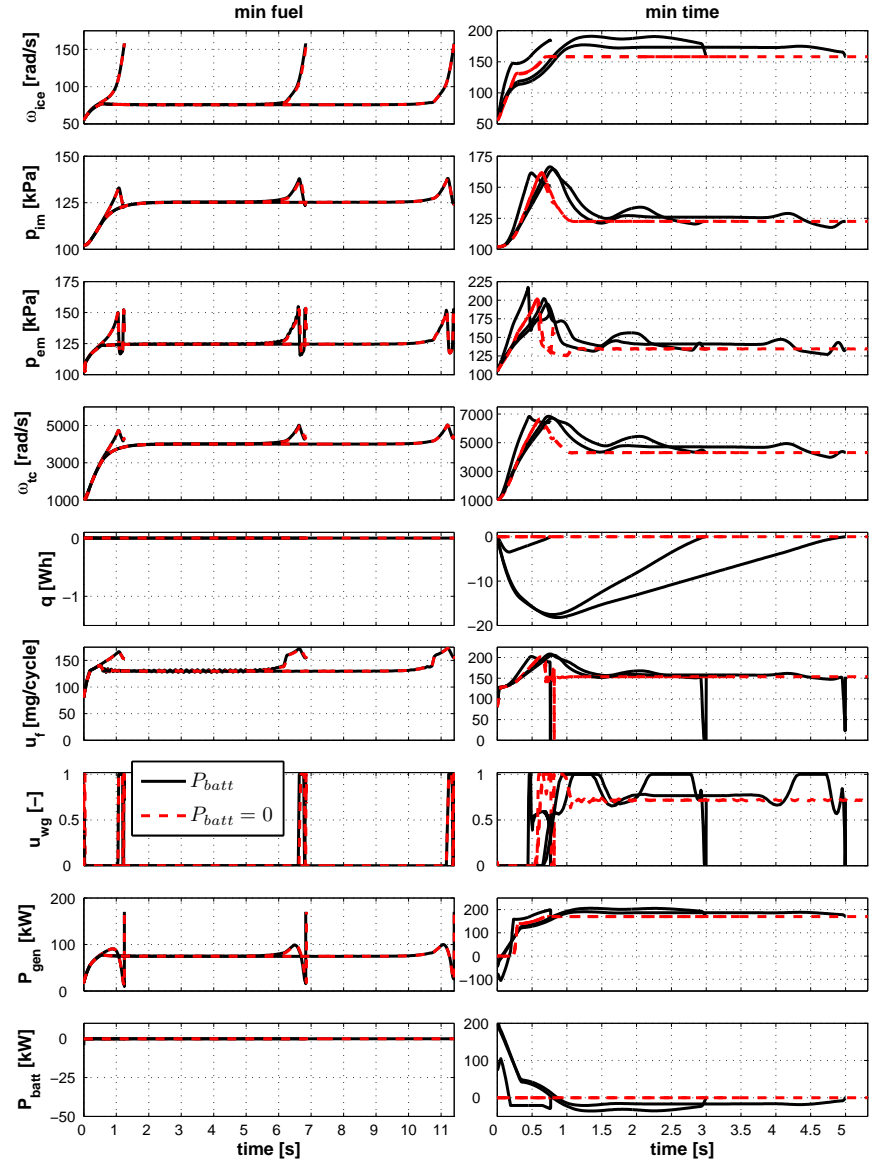

Fig. 5. Solutions for $E_{r e q}=\left[\begin{array}{ll}85 & 510 \\ 850\end{array}\right] \mathrm{kJ}$ with and without $P_{b a t t}=0$, Cont-lim.

Peak-lim only $E_{r e q}=85 \mathrm{~kJ}$ uses the energy storage, but with Power-lim all the solutions use the energy storage. Without energy storage these transients all approach the end operating point from a higher engine speed. With energy storage it is used to produce output power from the start. The generator power is also slightly lower than without energy storage, so is the maximum speed. The engine is thus accelerated to slightly higher engine speed, rotational energy that is then used to produce power. One reason for this is that when the generator then brakes the engine, since $P_{\text {out }} \leq P_{\text {req }}$ and $P_{\text {out }}=P_{\text {gen }}+$ $P_{b a t t}$ the case with energy storage can use $P_{g e n}>P_{r e q}$ and recharge the energy storage.

\section{B. Minimum time}

The minimum time solutions without the use of an energy storage also have similar characteristics. The optimal control is to accelerate the engine with wastegate closed up to roughly $130 \mathrm{rad} / \mathrm{s}$ and then a step in generator power to $P_{g e n}=P_{r e q}$ is applied. The engine then wanders towards the peak efficiency for that output power, within the given generator limits. This means that for Cont-lim the step is not to $P_{r e q}$, since that power is above the continuous limit, instead $P_{\text {gen }}$ follows the continuous limit up to the stationary point. When the end point is approached the wastegate is actuated to bring the engine to 

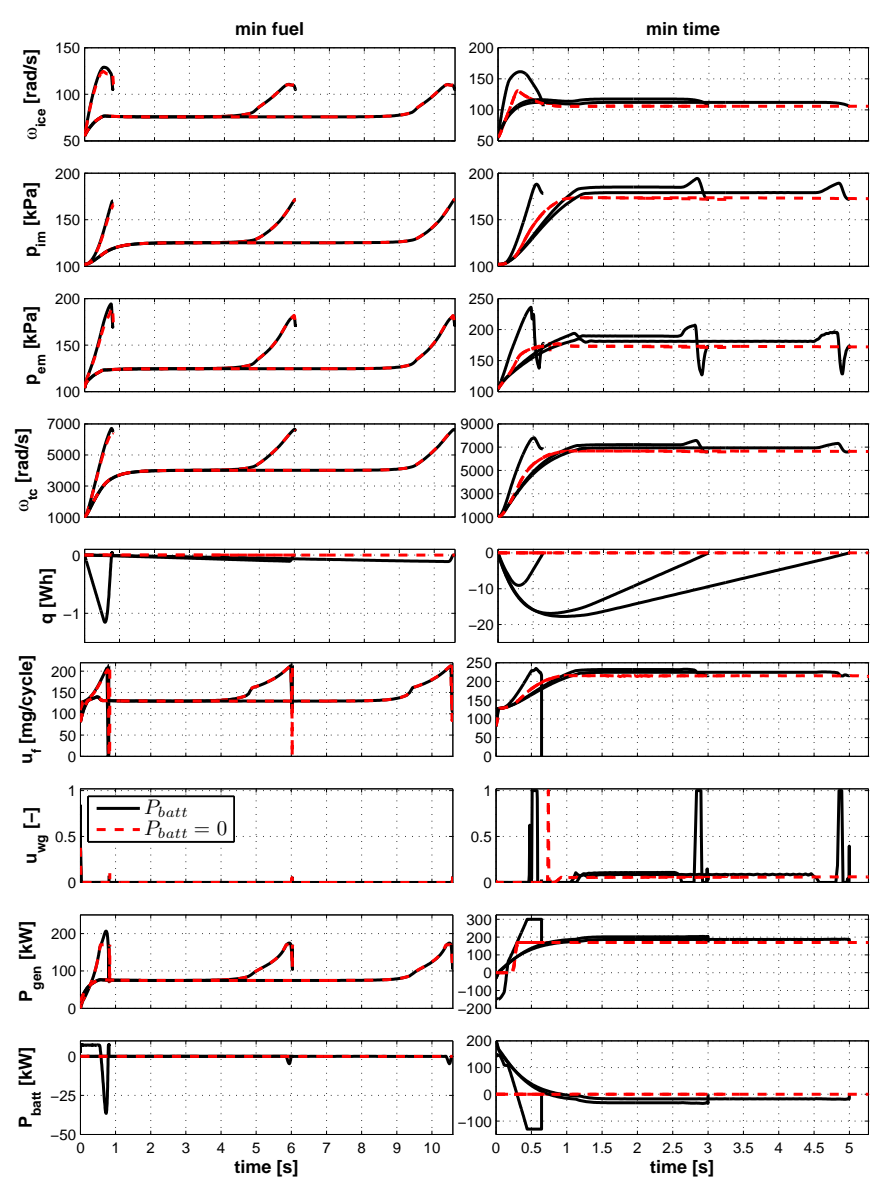

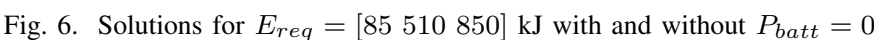
and Power-lim.

stationary conditions. For all limits except Standard-lim the stationary point is the same as the end point. For Standardlim the control is slightly different. This is since the peak efficiency is on the peak limit but the control has to end below the continuous limit. For larger $E_{r e q}$ it first wanders to the peak limit where it has a stationary phase before it accelerates to the end point, but for low $E_{r e q}$ it instead acclerates to the end point, without a stationary phase.

With energy storage and $E_{r e q}=85 \mathrm{~kJ}$ the generator is used in motoring mode for the first $0.17-0.19 \mathrm{~s}$, depending on generator limit, when it goes over into generator mode, producing power. Interesting to note is that the energy storage continues to produce output power, for all cases except Contlim, until approximately the top engine speed is reached. The generator power is then ramped up to the generator limit, which it follows until the end, whereas the engine follows the maximum torque line. During this phase the wastegate is actuated to maintain the engine torque within the limits while being on the smoke-limit. The transient then ends with an acceleration to meet the end constraints. Interesting to note is that for Peak-lim this end point is not in the region of peak efficiency, instead it is below the continuous limit.

For larger required energies the generator is only used in motoring mode for the first tenths of a second, instead the

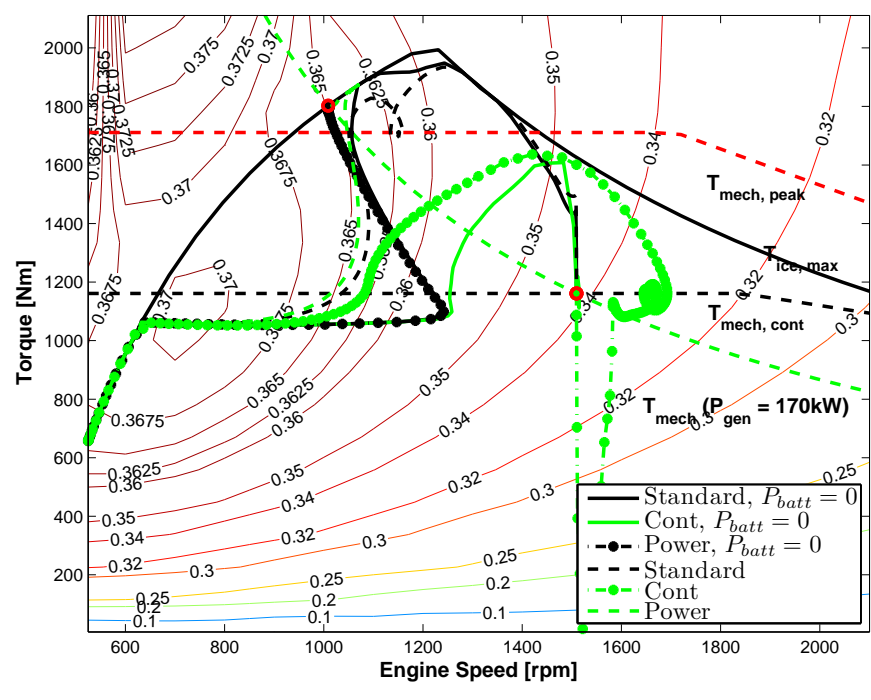

Fig. 7. Torque and Engine speed plot for the different limits, $\min T, E_{r e q}=$ $850 \mathrm{~kJ}$, with and without energy storage.

energy storage output is controlled so that the response time is immediate, i.e. $P_{\text {out }}=P_{r e q}$ from $\mathrm{t}=0$. For all but Cont-lim the generator is ramped up with a slight overshoot in engine speed before it approaches a stationary point recharging the energy storage. Cont-lim instead follows the generator limit to a stationary point. The stationary point for all cases is on the max generator limit, except Power-lim where it is on the engine max torque limit. At the stationary point the wastegate is actuated so the control follows the smoke-limit. The engine speed of the stationary point is $E_{r e q}$ dependent, the higher the $E_{r e q}$ the closer $P_{g e n}$ is to $P_{r e q}$ and thus the higher the efficiency, controlling the rate of charge of the energy storage. For larger energies there is more time to recharge the energy storage, which yields lower $P_{b a t t}$ and thus better efficiency of both the gen-set and the energy storage. The transient ends with the gen-set being controlled to end in the region of peak efficiency within the given limits.

\section{RESULTS}

The different criteria and limits, as well as the effects of the energy storage, are compared in Table III. In order for the results to be comparable, all results are augmented by holding the final controls until $850 \mathrm{~kJ}$ has been produced. There it is seen that even though the energy storage is used in some of the minimum fuel transients, the effect on the fuel consumption is negligible. The biggest effect is in changing the generator limits. Both the fuel economy and decrease in duration improves with increasing limits and if the entire range of the engine is allowed the improvement in both is roughly $10 \%$. Intersting to note is that even though all longer minimum fuel transients have a stationary phase at the peak efficiency of the gen-set, the decrease in fuel consumption of this is small. The increase in duration is however substantial.

For minimum time the decrease in fuel consumption without energy storage is almost as high as for minimum fuel for Peak and Power-lim. For the other two limits the fuel economy 


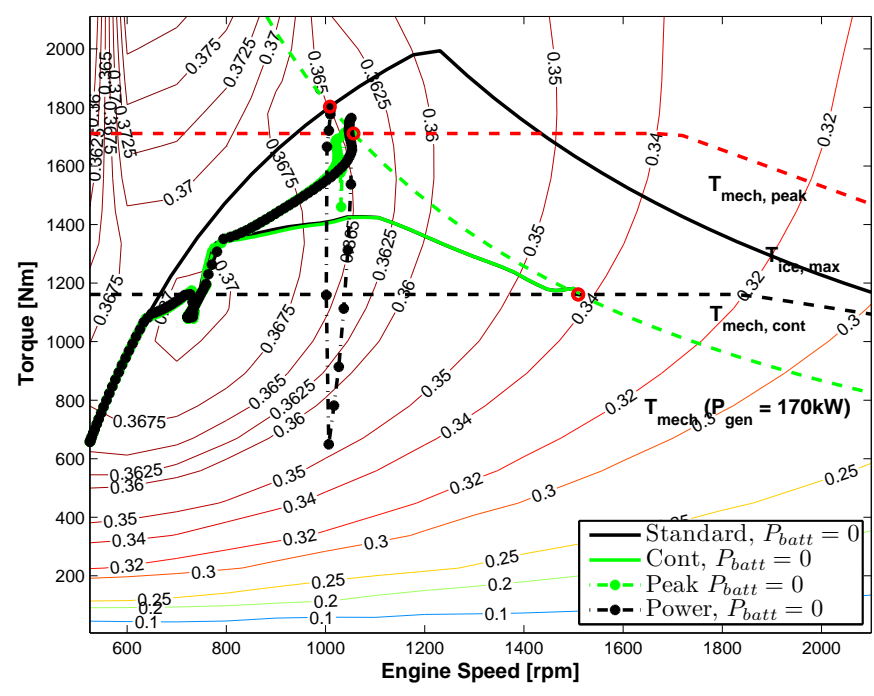

Fig. 8. Torque and Engine speed plot for the different limits, $\min m_{f}, P_{\text {batt }}=0, E_{\text {req }}=850 \mathrm{~kJ}$.

TABLE III

CHANGE IN FUEL CONSUMPTION AND DURATION OF THE DIFFERENT STRATEGIES COMPARED TO THE REFERENCE TRAJECTORY. $\Delta=\left[\Delta T[\%], \Delta m_{f}[\%], \Delta q_{\max }[W h]\right], \Delta q_{\max }=\max q-\min q$.

\begin{tabular}{|c|c|c|c|c|c|}
\hline \multicolumn{2}{|l|}{$\overline{E_{\text {req }}(T)}$} & \multicolumn{2}{|c|}{$\overline{\min } m_{f}$} & \multicolumn{2}{|c|}{$\overline{\min T}$} \\
\hline & & $P_{b a t t}=0$ & $P_{b a t t}$ & $P_{b a t t}=0$ & $P_{b a t t}$ \\
\hline & & \multicolumn{4}{|c|}{$\underline{\text { Standard-lim }}$} \\
\hline - & $\Delta$ & {$\left[\begin{array}{lll}0.2 & -0.7 & 0.0\end{array}\right]$} & {$\left[\begin{array}{lll}-0.1 & -0.7 & 0.5\end{array}\right]$} & {$\left[\begin{array}{lll}0.0 & 0.0 & 0.0\end{array}\right]$} & {$\left[\begin{array}{lll}-0.9 & -0.5 & 2.8\end{array}\right]$} \\
\hline $85 \mathrm{~kJ}$ & $\vec{\Delta}$ & {$\left[\begin{array}{lll}7.3 & -1.6 & 0.0\end{array}\right]$} & {$\left[\begin{array}{llll}7.2 & -1.6 & 0.0\end{array}\right]$} & {$\left[\begin{array}{lll}-1.3 & -0.2 & 0.0\end{array}\right]$} & {$\left[\begin{array}{llll}-3.0 & 1.1 & 6.7\end{array}\right]$} \\
\hline $510 \mathrm{~kJ}$ & $\Delta$ & {$\left[\begin{array}{lll}65.0 & -5.2 & 0.0\end{array}\right]$} & {$\left[\begin{array}{lll}65.0 & -5.2 & 0.0\end{array}\right]$} & {$\left[\begin{array}{lll}-1.7 & -4 & 0.0\end{array}\right]$} & {$\left[\begin{array}{lll}-6.7 & -2.5 & 16.3\end{array}\right]$} \\
\hline \multirow[t]{2}{*}{$850 \mathrm{~kJ}$} & $\bar{\Delta}$ & re & {$\left[\begin{array}{llll}1112.7 & -8.2 & 0.0\end{array}\right]$} & $.50 .0]$ & {$[-6.7-5$} \\
\hline & & \multicolumn{4}{|c|}{ Cont-lim } \\
\hline $85 \mathrm{k}$. & $\Delta$ & {$[7.3$} & {$\left[\begin{array}{lll}7.3 & -1.6 & 0.0\end{array}\right]$} & $.80 .0]$ & {$[-1.7$} \\
\hline $510 \mathrm{~kJ}$ & $\Delta$ & {$[65.2$} & {$\left[\begin{array}{llll}64.8 & -5.3 & 0.0\end{array}\right]$} & $20.0]$ & {$[-6.7 ?$} \\
\hline \multirow[t]{2}{*}{$850 \mathrm{~kJ}$} & $\Delta$ & {$\left[\begin{array}{lll}112.8 & -8.2 & 0.0\end{array}\right]$} & {$\left[\begin{array}{lll}1112.8 & -8.2 & 0.0\end{array}\right]$} & {$\left[\begin{array}{lll}-0.8 & -1.1 & 0.0\end{array}\right]$} & {$\left[\begin{array}{llll}-6.7 & 1.8 & 18.2\end{array}\right]$} \\
\hline & & \multicolumn{4}{|c|}{ Peak-lim } \\
\hline - & $\Delta$ & {$[0.8$} & {$\left[\begin{array}{lll}1.3 & -3.6 & 2.7\end{array}\right]$} & {$[0.0$} & {$[-1$.} \\
\hline $85 \mathrm{~kJ}$ & $\vec{\Delta}$ & {$[0.0-8$} & {$\left[\begin{array}{llll}0.1 & -8.8 & 0.4\end{array}\right]$} & $.70 .0]$ & {$\left[\begin{array}{llll}-3.2 & -2.2 & 6.1\end{array}\right]$} \\
\hline $510 \mathrm{~kJ}$ & $\Delta$ & {$[52.8$} & {$\left[\begin{array}{lll}52.8 & -9.7 & 0.0\end{array}\right]$} & $70.0]$ & {$\left[\begin{array}{lll}-6.7 & -7.2 & 16.3\end{array}\right]$} \\
\hline \multirow[t]{2}{*}{$850 \mathrm{~kJ}$} & $\Delta$ & {$\left[\begin{array}{lll}100.4 & -10.3 & 0.0\end{array}\right]$} & {$\left[\begin{array}{llll}100.5 & -10.3 & 0.0\end{array}\right]$} & {$\left[\begin{array}{lll}-1.7 & -8.7 & 0.0\end{array}\right]$} & {$\left[\begin{array}{lll}-6.7 & -7.5 & 17.0\end{array}\right]$} \\
\hline & & \multicolumn{4}{|c|}{ Power-lim } \\
\hline $85 \mathrm{~kJ}$ & $\Delta$ & 0 & {$\left[\begin{array}{llll}-0.9 & -8.9 & 1.2\end{array}\right]$} & $.70 .0]$ & {$[-4$} \\
\hline $510 \mathrm{~kJ}$ & $\Delta$ & {$\left[\begin{array}{lll}49.8 & -9.8 & 0.0\end{array}\right]$} & {$\left[\begin{array}{lll}49.9 & -9.8 & 0.1\end{array}\right]$} & {$\left[\begin{array}{lll}-1.7 & -8.9 & 0.0\end{array}\right]$} & {$\left[\begin{array}{lll}-6.7 & -7.8 & 16.9\end{array}\right]$} \\
\hline $850 \mathrm{~kJ}$ & $\Delta$ & {$\left[\begin{array}{lll}97.5 & -10.4 & 0.0\end{array}\right]$} & {$\left[\begin{array}{lll}97.6 & -10.4 & 0.1\end{array}\right]$} & {$\left[\begin{array}{lll}-1.7 & -8.9 & 0.0\end{array}\right]$} & {$\left[\begin{array}{lll}-6.7 & -7.9 & 17.7\end{array}\right]$} \\
\hline
\end{tabular}

potential is however a bit more limited. Adding an energy storage decreases the fuel gains but instead it improves the response time. For $E_{r e q} \geq 510 \mathrm{~kJ}$ the response time is immediate, regardless of generator limit, and this with an energy storage of only up to $18 \mathrm{Wh} \approx 65 \mathrm{~kJ}$.

\section{CONCLUSION}

The effect of the generator limit on the optimal control of a diesel-electric powertrain with and without the use of an energy storage to assist during the transients is studied. In order to just study the transients the charge in the energy storage is required to be the same at start and end. For minimum fuel problems the energy storage remains virtually unused. The control consists of two phases, first it accelerates to the region of peak efficiency for the gen-set where it stays until the end of the transient where it accelerates to meet the end constraints. The gain of staying at the region of peak efficiency is however small. Comparing different horizon lengths, the increase in fuel consumption of meeting the drivers request after $0.5 \mathrm{~s}$ instead of $5 \mathrm{~s}$ is less than $1 \%$ for Peak and Power-lim. For the cases where the control has to end below the continuous limit the difference is larger, since the efficiency of that operating point is substantially lower.

For minimum time the energy storage is used both to accelerate the engine and to produce output power. For larger $E_{r e q}$ the solution has a stationary phase on one of the limits, either of the generator or of the engine, depending on the case, both with and without energy storage. With energy storage this stationary point is higher in engine speed since the energy storage has to be recharged. This results in a slightly higher consumption compared to the case without energy storage, but for horizons of $510 \mathrm{~kJ}$ and higher the consumption still is lower than the reference trajectory, and faster.

The fuel economy for the minimum fuel formulation improves with increasing $E_{r e q}$, but the time penalty for this quite quickly becomes relatively large. The minimum fuel solution stays in the region of peak efficiency for a large part of the transient, producing a power lower than the requested, something that might not be acceptable. It however produces power from the start. The minimum time solutions first accelerate the engine before it starts to produce power. For longer energy horizons, this response time is small, roughly tenths of a second before it reaches the requested power. This could still be perceived as strange. It is seen that adding a small energy storage can reduce this delay, and even remove it completely for larger requested energies, regardless of generator limits, with an energy storage of just $65 \mathrm{~kJ}$. Also the minimum time formulation not only decreases the response time of the powertrain, it also decreases fuel. For the case without energy storage, and Peak or Power-lim, the minimum time controls just increase the consumption with roughly $1 \%$ compared to minimum fuel, despite being substantially faster.

\section{ACKNOWLEDGMENT}

The support from BAE Systems in Örnsköldsvik and the funding from the Swedish Energy Agency FFI is gratefully acknowledged.

\section{REFERENCES}

[1] M. Sivertsson and L. Eriksson, "Time and fuel optimal power response of a diesel-electric powertrain,' in E-COSM'12 - IFAC Workshop on Engine and Powertrain Control, Simulation and Modeling, Paris, France, October 2012.

[2] - "Optimal step responses in diesel-electric systems," in Mechatronics'12 - The 13th Mechatronics Forum International Conference, Linz, Austria, September 2012.

[3] — "Optimal transient control and effects of a small energy storage for a diesel-electric powertrain," in AAC'13-The 7th IFAC Symposium on Advances in Automotive Control, Tokyo, Japan, September 2013.

[4] M. Benz, M. Hehn, C. H. Onder, and L. Guzzella, "Model-based actuator trajectories optimization for a diesel engine using a direct method,' Journal of Engineering for Gas Turbines and Power, vol. 133, no. 3, pp. 298-312, 2011.

[5] T. Nilsson, A. Fröberg, and J. Åslund, "Optimal operation of a turbocharged diesel engine during transients," in SAE Technical Paper 201201-0711, Detroit, Michigan, USA, 2012.

[6] J. Wahlström and L. Eriksson, "Modelling diesel engines with a variablegeometry turbocharger and exhaust gas recirculation by optimization of model parameters for capturing non-linear system dynamics," Proceedings of the Institution of Mechanical Engineers, Part D, Journal of Automobile Engineering, vol. 225, no. 7, pp. 960-986, 2011. 